\title{
Investigation of Anisotropic Cylindrical Semiconductor-Dielectric Waveguides
}

\author{
Darius Plonis $^{1}$, Vacius Malisauskas ${ }^{1}$, Andrius Katkevicius ${ }^{1}$ \\ ${ }^{1}$ Department of Electronic Systems, Vilnius Gediminas Technical University, \\ Naugarduko St. 41-413, LT-03227 Vilnius, Lithuania \\ darius.plonis@vgtu.lt
}

\begin{abstract}
${ }^{1}$ Abstract-The use of semiconductor-dielectric waveguides is low at the moment due to disadvantage of high attenuation of electromagnetic microwaves. The attenuation depends on structures and materials of semiconductor-dielectric waveguides. Dependences of transmission coefficient on the frequency of semiconductor-dielectric waveguides and on different densities of electrons in semiconductors are studied in this paper. As well as the influence of periodicity on transmission of electromagnetic microwaves in semiconductordielectric waveguides is discussed. It is shown that frequency dependences of transmission coefficient of periodical semiconductor-dielectric waveguides obtain the properties of the band pass filter. Also it is shown that the choice of semiconductor and dielectric materials have a significant impact on the nature of the transmission and attenuation of electromagnetic microwaves.
\end{abstract}

Index Terms-Electromagnetic propagation, electrons, charge carrier density and mobility, periodic structures.

\section{INTRODUCTION}

Various waveguides systems are widely applied for travelling-wave tubes, delay lines, phase modulators or converters and other microwave devices [1]-[5].

On the other hand the use of semiconductor waveguides is low at the moment. Other authors have shown what attenuation of electromagnetic (EM) microwaves in semiconductor waveguides is high [6]. High attenuation of EM microwaves reduces possibilities to use semiconductor waveguides in microwave systems.

For instance $\mathrm{SiC}$ semiconductor waveguides could be used as microwave phase shifters due to high phase conversion range of EM microwaves. The temperature controllable waveguides without external dielectric could be manufactured from dipolar glass but the use of these materials does not reduce the high attenuation of EM microwaves [7].

The Gauss pulses distortion in layered cylindrical dielectric waveguide is investigated by I. O. Vardiambasis [8]. The analysis of waveguides is performed when dielectrics are ideal without losses. Structures of EM fields in dielectric waveguides with anisotropic walls are investigated in paper [9]. The transmission and reflection coefficients of periodic ferrite semiconductor waveguides are discussed in papers [10]-[12].

Application of the strong lossy-dielectric loaded periodic

Manuscript received March 24, 2015; accepted June 27, 2015. waveguide in millimeter-wave gyro travelling microwave tube is described in paper [13]. Dielectric-loaded metal cylindrical waveguide for applications of gyrotron-travelingwave amplifier is discussed in paper [14].

The relevant scientific problem of development and applications of electrodynamical models (further models) of gyrotropic devices is partially addressed in this paper. The problem is solved by using models of open cylindrical, generalized circular cross section, layered semiconductordielectric (gyroelectric) waveguides. Characteristics of microwave which propagates in these waveguides are obtained by applying the method of numerical experiment (modelling). Analogical models of ferrite-dielectric (gyromagnetic) waveguides are used occasionally. These models are investigated using the methods of numerical and physical experiments. This paper is part and continuation of our other scientific works on this topic [15], [16].

Gyrotropy is a type of anisotropy and is characterized by isotropic material which experience artificial anisotropy by operating under constant magnetic field [15].

Gyrotropic devices are often used in microwave range: phase shifters and modulators, polarizers and circulators, elements of antennas and so on. Anisotropic ferrite-dielectric devices are well investigated, but their control is limited to a magnetic field. Anisotropic semiconductor-dielectric waveguides are less investigated, but their control capabilities are significantly higher. Semiconductordielectric waveguides can be controlled by the magnetic field, temperature, infrared rays, visible light, $\gamma$-rays. Therefore it's now important to investigate semiconductordielectric waveguides by comparing their research methods and electrodynamical properties with ferrite-dielectric waveguides.

There are problems encountered in anisotropic waveguide's modelling and material's selection by investigating and applying anisotropic devices. Ferrite and semiconductor cores are hard and brittle therefore cores usually have the shape of cylindrical circular cross section rods. Models with external semiconductor or dielectric layers can be applied in order to modify the electrodynamical properties and increase resistance of semiconductor cores. The choice of ferrites is small but they are distinguished by low attenuation of propagating waves in microwave devices. A large choice of semiconductors is determined by the used materials, the conductivity types of materials and densities of free carriers 
$\left(i, n^{-}, n, n^{+}, p^{-}, p, p^{+}\right)$, mobility of carriers and effective mass, constant of crystal lattice, the relative permittivity and others.

The aim of this paper is to investigate dependences of transmission coefficient on the frequency of non-periodical and periodical models of semiconductor-dielectric waveguides (SDWs) on different temperatures and densities of electrons in semiconductors as well as to investigate possibilities of applications of such models.

Non-periodical layered ferrite and semiconductordielectric waveguides are naturally anisotropic in crosssection. The same type periodical waveguides are naturally anisotropic in cross-section and longitudinal-view. These waveguides are composed of several different materials and waveguide's properties are different in different directions.

Tasks which are addressed in this paper: the improvement of electrodynamical models of semiconductor-dielectric waveguides; development and investigation of computer models of layered SDWs using a commercial software CST Microwave Studio ${ }^{\circledR}$ [17]; the investigation of possibilities of SDWs applications in microwave devices.

Methods used at work: commercial software CST Microwave Studio ${ }^{\circledR}$ is dedicated to the development of computer models of SDWs, calculation of parameters of SDWs and comparison of their parameters; hybrid numerical methods which are used in CST Microwave Studio ${ }^{\circledR}$ software divide the analysed area into the elements; testing, physical and numerical experiments (modelling) are dedicated to the investigation and verification of parameters and characteristics of models of SDWs.

There were created new computer models of nonperiodical and periodical SDWs and obtained results of their numerical experiments using modern commercial software.

\section{THE ELECTRODYNAMICAL MODEL}

In this chapter is presented electrodynamical model of periodical semiconductor-dielectric waveguides which is used for analysis of non-periodical and periodical SDWs. For verification of modelling results are used electrodynamical models which are presented in our previous works [15], [16], [18].

Dependences of transmission coefficients of propagating microwaves in SDWs versus frequency are calculated in $8 \mathrm{GHz}-80 \mathrm{GHz}$ frequency range in this paper. This frequency range corresponds to the $37.5 \mathrm{~mm}-3.75 \mathrm{~mm}$ length microwave range in the air. The length of propagating microwaves in SDW is from $9.7 \mathrm{~mm}$ to $0.1 \mathrm{~mm}$ taking into account the relative permittivity which is approximately equal to 15 and permeability which is approximately equal to 1 of semiconductors and dielectrics which have been used. Transversal and longitudinal dimensions of the investigated SDWs are in the row of length of microwave. Therefore SDWs are electrodynamical systems with distributed parameters. The dielectric and magnetic permeabilities, diameters and lengths are not only structural SDWs parameters, but, most importantly, electrodynamical parameters as well.

It is difficult to perform the physical experiment due to the relatively small diameter of SDW, long lengths, hardness and fragility of material, cylindrical circular cross section shape, complex construction, monocrystal semiconductor structure and the low density of free carriers $10^{19} \mathrm{~m}^{-3}$ $10^{20} \mathrm{~m}^{-3}$ which is influenced by microwave attenuation. Semiconductor monocrystals are produced by the zone smelting or pulling from the melt methods. Quite large $150 \mathrm{~mm}-300 \mathrm{~mm}$ in diameter and $1.5 \mathrm{~m}-2 \mathrm{~m}$ long rods are sanded smooth and sliced into $0.4 \mathrm{~mm}-0.8 \mathrm{~mm}$ thick plates. These dimensions and cross-sectional shape are not compatible with the SDW structure and design. Diffusion of impurities, implantation of ion is carried out to a depth of a few micrometers. The thickness of epitaxial layers, dielectric layers is also up to several micrometers. Density of impurities in typical semiconductor devices is about $10^{19} \mathrm{~cm}^{-3}-10^{20} \mathrm{~cm}^{-3}$, so $10^{6}$ times higher than permissible in SDW. Therefore we were unable to obtain adequate samples and had to be confined to the numerical experiment (modelling).

\section{A. The Electrodynamical Model of Periodical Semiconductor-dielectric Waveguides}

The cross-section of electrodynamical model of open cylindrical semiconductor-dielectric waveguide with periodical layer is presented in (Fig. 1(a)).

The model consists of several layers: 1 - semiconductor core (parameters upper index is ' $\mathrm{s}$ '), 2 - semiconductor or semiconductor-air layer, 3 - external dielectric layer, 4 - air.

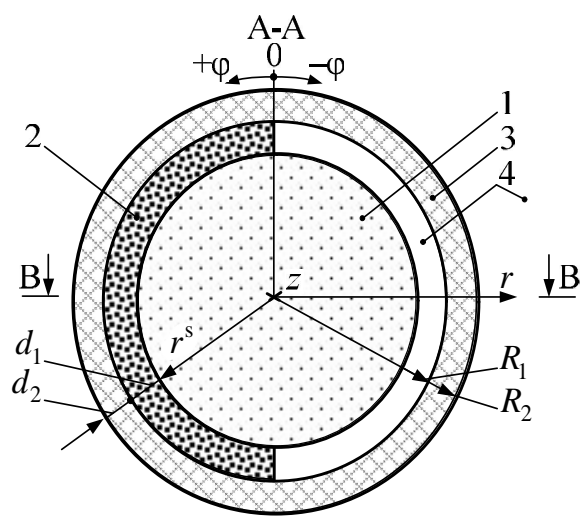

(a)

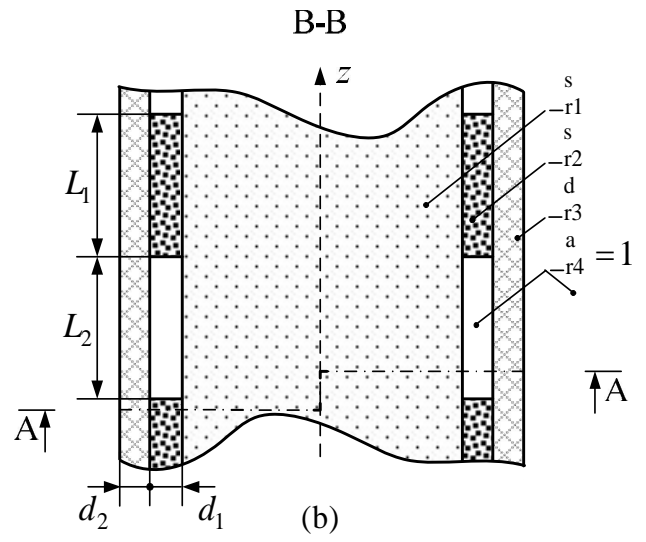

Fig. 1. The electrodynamical model of open cylindrical semiconductordielectric waveguide with periodical semiconductor layer: (a) cross-section; (b) longitudinal-view.

The complex permittivity of semiconductor core and external semiconductor layer (rings) could be calculated by using cold-plasma expression 


$$
\varepsilon_{\mathrm{r}}^{\mathrm{s}}=\varepsilon_{\mathrm{k}}^{\mathrm{sn}}-\frac{\omega_{\mathrm{p}}^{2}}{\omega(\omega-\mathrm{i} \zeta)}
$$

where $\varepsilon_{\mathrm{k}}^{\mathrm{sn}}$ is the $n$-type semiconductor dielectric constant; $\omega_{\mathrm{p}}$ is the angular plasma frequency; $\omega=2 \pi f$ is the angular frequency; $f$ is the frequency of EM microwaves; $\zeta$ is the electrons total collision frequency with semiconductor lattice constant. The semiconductor core radius is $r^{\mathrm{s}}=1 \mathrm{~mm}$.

External layers are characterized by complex permittivities $\underline{\varepsilon}_{\mathrm{r} 2}^{\mathrm{s}}$ and $\underline{\varepsilon}_{\mathrm{r} 3}^{\mathrm{d}}$ respectively, which widths are $d_{1}$ and $d_{2}$. Radii of external layers are $R_{1}$ and $R_{2}$.

The longitudinal-view of electrodynamical model of open cylindrical SDW with periodical semiconductor-dielectric layers is presented in (Fig. 1(b)).

The analysis of the model of periodical SDW was performed by taking into account that waveguide consists of six semiconductor rings, which lengths are equal to $L_{1}=5 \mathrm{~mm}$, and lengths of air rings are equal to $L_{2}=5 \mathrm{~mm}$. The overall length of the model is $L=55 \mathrm{~mm}$. Thicknesses of external layers were increased in order to obtain properties of microwave filter in models of periodical SDWs. Relative thicknesses are selected $d_{1} / r^{\mathrm{s}}=0.3$ and $d_{2} / r^{\mathrm{s}}=0.2$.

The periodical waveguides could be transformed in to non-periodical waveguides, when second layer of the waveguide is continuous and it is made from semiconductor or air, and the boundary between rings disappears, when permittivities of rings will be the same.

\section{B. Verification of Modelling Results of Waveguides}

For verification of physical and numerical experiment's results was selected model of open waveguide of $1 \mathrm{SCh} 11$ ferrite. Experimental investigations of ferrite waveguides were carried out in company JSC "Elmika". The permittivity of $1 \mathrm{SCh} 11$ ferrite was equal to $\varepsilon_{\mathrm{r}}^{\mathrm{f}}=14.6$ and the tangent of the loss angle was $\tan \delta=610^{-4}$, and complex relative permeability of waveguide is equal to ${ }_{-\mathrm{r}}^{\mathrm{f}}=1-\mathrm{i} 5 \cdot 10^{-3}$.

Measurements were performed with a R2400 scalar analyser which was produced in the company JSC "Elmika". Limits of measurement of R2400 scalar analyser are equal to $25 \mathrm{GHz}-40 \mathrm{GHz}$ frequency range, 1.1-5.0 standing microwave ratio and transmission coefficient's $0 \mathrm{~dB}-35 \mathrm{~dB}$ range. Results of physical experiments were compared with the results of numerical experiments which were obtained using the CST Microwave Studio ${ }^{\circledR}$ software package.

The research structure of model ferrite waveguides is presented in Fig. 2. EM microwave generator generates the main type $\mathrm{H}_{10}$ microwave in the rectangular waveguide. Ferrite isolator spreads the wave only in one direction. The first coupler directs the main type microwave to the analyser and to the second coupler. The second coupler directs the microwave into investigated waveguide. The reflected microwave from the investigated waveguide is directed to the R2400 scalar analyser through the second coupler.

The third coupler is used to transfer microwaves to the R2400 scalar analyser and to the load. Microwaves are reinforced in amplifiers before reaching the scalar analyser.
Scalar analyser and EM microwave generator are managed by personal computer (PC). Research results are visualized using the PC.

The longitudinal-view and cross-section of $1 \mathrm{SCh} 11$ ferrite waveguides model is presented in Fig. 3. The length of the investigated waveguide rejecting the conical ends is equal to $L_{1}=40.2 \mathrm{~mm}$, the length of conical ends $L_{0}=22.5 \mathrm{~mm}$, the overall length of the waveguide is equal to $L=L_{1}+2 L_{0}=85.2 \mathrm{~mm}$, the radius of the waveguide $r^{\mathrm{f}}=1.1 \mathrm{~mm}$.

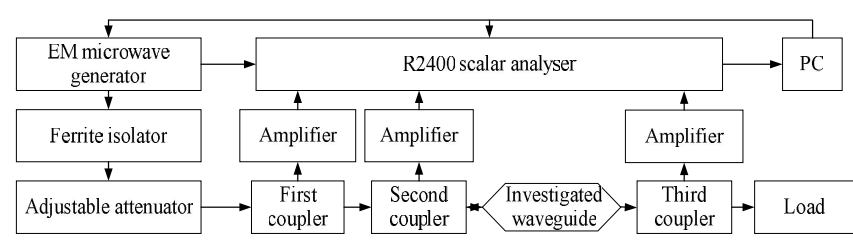

Fig. 2. Research structure of ferrite waveguides model

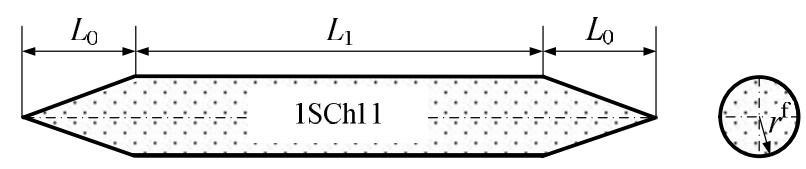

Fig. 3. The longitudinal-view and cross-section of 1 SCh11 ferrite waveguides model.

Dependences of transmission coefficient on the frequency of propagating microwaves in ferrite waveguides obtained using physical and numerical experiments are presented in Fig. 4. It can be seen that dependences of transmission coefficient on the frequency of propagating microwaves in 1SCh11 ferrite waveguides obtained using physical experiment are similar to dependences obtained using commercial software.

Results of physical and numerical experiments are similar. Resonance is in the frequency range from $32 \mathrm{GHz}$ to $38.8 \mathrm{GHz}$ due to couplers and incompatibility of characteristic impedances in the investigated waveguide Fig. 4.

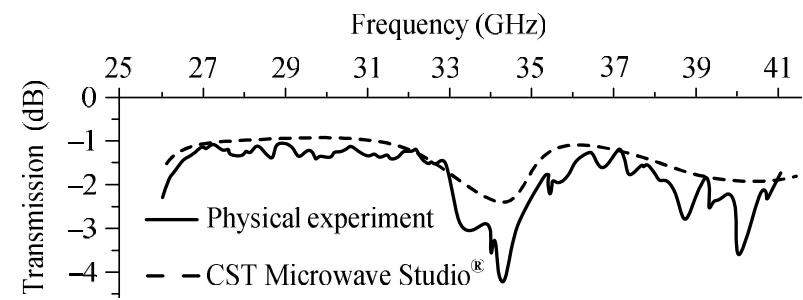

Fig. 4. Physical and numerical experiments dependences of transmission coefficient on the frequency of propagating microwaves in ferrite waveguides model.

Part of the EM microwaves are reflected from the ends of investigated waveguide therefore the attenuation suddenly increases in the resonance frequency range. The resonance appears in the same frequency range and in computer simulation, but in this case the distortion of characteristics is lower. The transmission coefficient in the resonance frequency range at $34.1 \mathrm{GHz}$ frequency is about 2 times larger during the physical experiment then in modelling.

Dependences of attenuation coefficients on the frequency of propagating $\mathrm{HE}_{11}$ microwaves in ferrite $1 \mathrm{SCh} 11$ waveguides model are presented in Fig. 5. 
Since it is impossible to measure attenuation coefficients of propagating microwaves physical experiment and comparison of results is carried out in order to find differences between the numerical experiments. Therefore the comparison is carried out of results which were obtained using the author's algorithm [18] and commercial software.

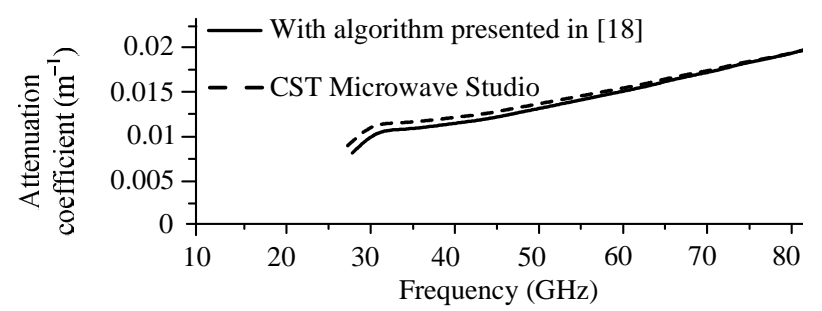

Fig. 5. Dependences of attenuation coefficients on the frequency of propagated $\mathrm{HE}_{11}$ microwaves in ferrite waveguides model.

The major differences between results are in the lower frequency side Fig. 5. Differences seek up to $9 \%$ and decreases until it reach the $0.1 \%$ level because of increasing frequency of EM microwaves. The results of physical and numerical experiments varies no more than two times. However the accuracy of comparable characteristics remained unknown in both cases.

\section{THE ANALYSIS OF MODELS OF NON-PERIODICAL SEMICONDUCTOR-DIELECTRIC WAVEGUIDES}

Several models of non-periodical SDWs are discussed in this chapter. The structures of the electrodynamical models are: $n$-InSb core and $n$-SiGe, TM-15 layers; $n$-SiGe core and $n$-InSb, TM-15 layers; $n$-GaAs core and $n$-InAs ${ }_{1-x} \mathrm{Sb}_{x}$, TM-15 layers. The TM-15 dielectric is used for the external layer. Its complex permittivity is $\underline{\varepsilon}_{\mathrm{r} 3}^{\mathrm{d}}=15\left(1-\mathrm{i} \cdot 10^{-4}\right)$.

The analysis of models of SDWs is performed by taking in account density of electrons $N$, semiconductor electron mobility $\mu$, effective mass $m^{*}$ and temperature $T$. Values of following parameters are selected based on the literature [19], [20].

The model of non-periodical waveguide which structure consist of $n$-InSb core and $n$-SiGe, TM-15 layers is investigated, when external temperature is $T=300 \mathrm{~K}$ and densities of electrons are $N=10^{19} ; 510^{19} ; 10^{20} \mathrm{~m}^{-3}$. The length of waveguide is $L=55 \mathrm{~mm}$ and normalized widths of external layers are $d_{1} / r^{\mathrm{s}}=d_{2} / r^{\mathrm{s}}=0.15$.

Frequency dependences of transmission coefficient of propagated $\mathrm{HE}_{11}$ mode of above discussed waveguide model are presented in Fig. 6.

The SDW has a lower attenuation of EM microwaves when the density of electrons is $N=10^{19} \mathrm{~m}^{-3}$. Transmission characteristics of EM microwaves of the waveguide are similar to characteristics of the high pass filter. The highest attenuation of EM microwaves could be received when densities of electrons are equal to $N=510^{19} ; 10^{20} \mathrm{~m}^{-3}$.

The highest attenuation of EM microwaves is at $45 \mathrm{GHz}$. Since the energy of EM microwaves is absorbed and above discussed features are maintained the SDW could be used for manufacture of attenuators and absorbers of EM microwaves when densities of electrons are equal to $N=510^{19} ; 10^{20} \mathrm{~m}^{-3}$.
The attenuation of EM microwave could be decreased by changing the type of semiconductor. Frequency dependences of transmission coefficient of propagated modes of SDW which core is from $n$-SiGe semiconductor, first external layer is from $n$-InSb semiconductor and second external layer is from TM-15 dielectric are presented in Fig. 7.

The electron mobility is 17.5 times lower in $n$-SiGe than in $n$-InSb. The dielectric constants are almost the same for both semiconductors. The effective mass is $\sim 114$ times higher in $n$-SiGe than in $n$-InSb. The electron mobility has the greatest influence on variation of attenuation of EM microwaves comparing with other parameters.

From analysis of dependences of transmission coefficient on the frequency (Fig. 6 and Fig. 7) it's seen, that attenuation of EM microwaves is lower in model of nonperiodical SDW which structure consist of $n$-SiGe core and $n$-InSb, TM-15 layers than in model of waveguide which structure consist of $n$-InSb core and $n$-SiGe, TM-15 layers. EM microwaves are more concentrated in semiconductor core in models of multilayer semiconductor waveguide.

Frequency dependences of transmission coefficient of model of non-periodical waveguide, which structure consist of $n$-GaAs core and $n$-InAs ${ }_{1-x} \mathrm{Sb}_{x}, \mathrm{TM}-15$ layers are presented in Fig. 8. The density of electrons is $N=10^{19} \mathrm{~m}^{-3}$ and external temperature is varied from $125 \mathrm{~K}$ to $200 \mathrm{~K}$.

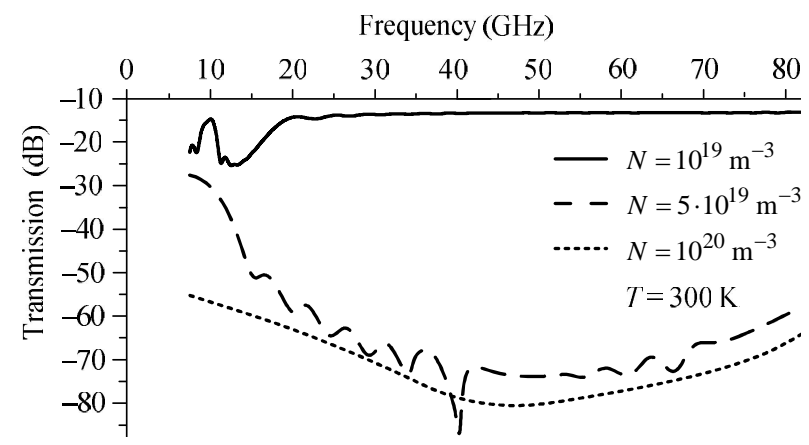

Fig. 6. Propagated HE11 mode's transmission coefficient's frequency dependences of non-periodical semiconductor-dielectric waveguides models which consist of $n$-InSb core and $n$-SiGe, TM-15 layers.

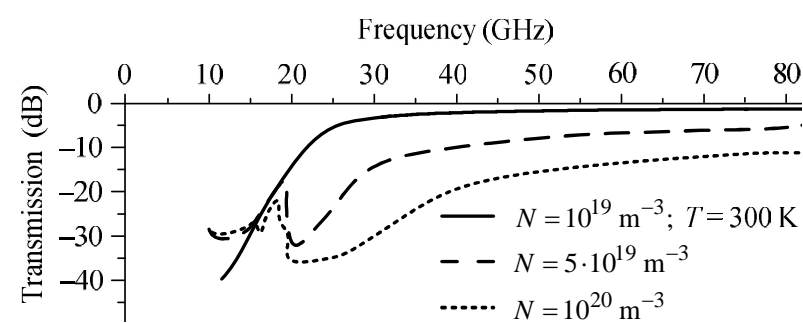

Fig. 7. Propagated $\mathrm{HE}_{11}$ mode's transmission coefficient's frequency dependences of non-periodical semiconductor-dielectric waveguides models which consist of $n$-SiGe core and $n$-InSb, TM-15 layers.

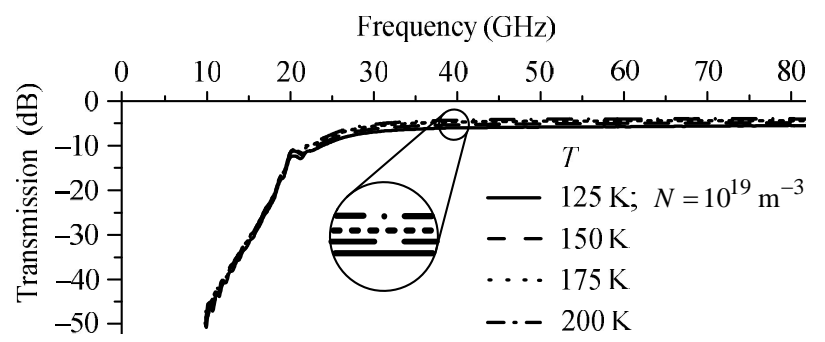

Fig. 8. Propagated HE 11 mode's transmission coefficient's frequency dependences of non-periodical semiconductor-dielectric waveguides models which consist of $n$-GaAs core and $n$-InAs $1_{-x} \mathrm{Sb}_{x}$, TM-15 layers. 
The attenuation of EM microwaves is stable in frequency range from $30 \mathrm{GHz}$ to $80 \mathrm{GHz}$ (Fig. 8). The attenuation of EM microwaves is lower, when external temperature is $T=200 \mathrm{~K}$.

After the analysis of electrodynamical models of non-periodical SDWs it can be concluded, that lower attenuation of EM microwaves could be received in models of waveguides which structure consist of $n$-SiGe core and $n$-InSb, TM-15 layers. However the attenuation of EM microwaves could be controlled only in models of waveguides which structure consist of $n$-GaAs core and $n$-InAs ${ }_{1-x} \mathrm{Sb}_{x}, \mathrm{TM}-15$ layers.

\section{THE ANALYSIS OF MODELS OF PERIODICAL SEMICONDUCTOR-DIELECTRIC WAVEGUIDES}

The analysis of electrodynamical models of periodical SDWs which structure consist of $n$-SiGe core and $n$-InSbair, TM-15 layers is performed, when densities of electrons are $N=10^{19} ; 10^{20} \mathrm{~m}^{-3}$. These densities are selected in order to reveal the influence of propagating $\mathrm{HE}_{11}$ modes.

Dependences of transmission coefficient on the frequency of $\mathrm{HE}_{11}$ mode propagating in models of periodical SDWs are similar to characteristics of the band pass filter (Fig. 9). The best characteristics of band pass filter are achieved, when density of electrons is $N=10^{19} \mathrm{~m}^{-3}$. The attenuation of EM microwaves in waveguides increases and properties of band pass filter decrease when density of electrons increases.

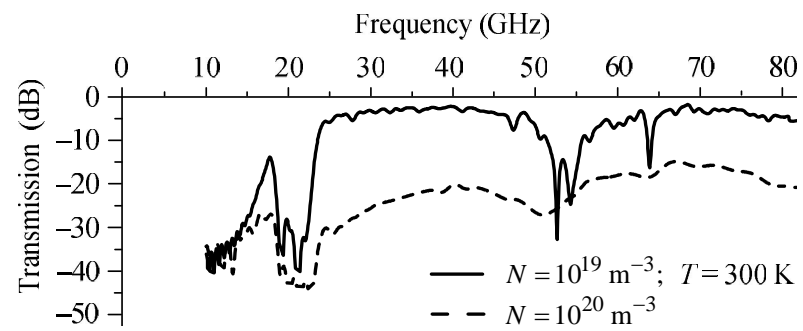

Fig. 9. Propagated $\mathrm{HE}_{11}$ mode's transmission coefficient's frequency dependences of periodical semiconductor-dielectric waveguides models which consist of $n$-SiGe core and $n$-InSb-air, TM-15 layers.

Ten times higher density of electrons $N=10^{20} \mathrm{~m}^{-3}$ increases attenuation of EM microwaves approximately $20 \mathrm{~dB}$ and decreases resonances in all analysed frequency range. Resonances appear when EM microwaves reflect from the edges of semiconductor rings (Fig. 9).

Frequency dependences of transmission coefficient of propagated $\mathrm{HE}_{11}$ mode in model of periodical SDWs which structure consist of $n$-GaAs core and $n$-InAs ${ }_{1-x} \mathrm{Sb}_{x}$-air, TM-15 layers are presented in Fig. 10. The analysis of this model is performed by taking in to account the density of electrons $N=10^{19} \mathrm{~m}^{-3}$ and temperatures $T=125 ; 200 \mathrm{~K}$. The attenuation of EM microwaves decreases, when temperature increases till $200 \mathrm{~K}$. The stop band is visible and the width of the stop band is from $43 \mathrm{GHz}$ to $52 \mathrm{GHz}$.

The highest attenuation of EM microwaves is in the middle of the stop band and it is equal to $-45 \mathrm{~dB}$.

The attenuation of the $\mathrm{HE}_{11}$ mode could be adjusted by changing temperature $T$. Therefore these models of waveguides are more suitable than waveguides which structure consist of $n$-SiGe core and $n$-InSb-air, TM-15 layers.

Comparing (Fig. 9) and (Fig. 10) it's seen, that the attenuation of $\mathrm{HE}_{11}$ mode is $-4 \mathrm{~dB}$ in frequency range from $24 \mathrm{GHz}$ to $47 \mathrm{GHz}$, when density of electrons is $N=10^{19} \mathrm{~m}^{-3}$. The attenuation of EM microwaves is obtained in models of SDWs which structure consist of $n$-SiGe core and $n$-InSb, TM-15 layers.

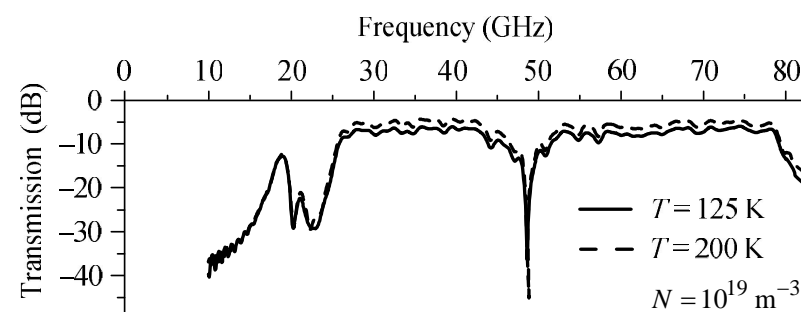

Fig. 10. Propagated $\mathrm{HE}_{11}$ mode's transmission coefficient's frequency dependences of periodical semiconductor-dielectric waveguides models which consist of $n$-GaAs core and $n$-InAs1-x $\mathrm{Sb}_{x}$-air, TM-15 layers.

The attenuation of EM microwaves in models of SDWs which structure consist of $n$-GaAs core and $n$-InAs ${ }_{1-x} \mathrm{Sb}_{x}$, TM-15 layers is $-5 \mathrm{~dB}$ at frequency range from $25 \mathrm{GHz}$ to $43 \mathrm{GHz}$ and temperature $T=200 \mathrm{~K}$. But the width of the stop band is smoother in this type of waveguides in comparison with other waveguides.

From analysis of dependences of transmission coefficient on the frequency it's seen, that characteristics are not smother. It could be explained by the fact that semiconductor rings change impedances of the waveguides.

Impedances of waveguides could be combined with air space by changing the edges of semiconductor rings. The edges of semiconductor rings should be conical. These changes of structures of models of periodical SDWs are presented in Fig. 11. The lengths of conical edges of periodical rings are $L=0.5 \mathrm{~mm}$ and the semiconductor layer thickness is $d_{1}=0.3 \mathrm{~mm}$.

Frequency dependences of transmission coefficient of propagated $\mathrm{HE}_{11}$ mode in models of periodical SDWs with conical edges of semiconductor rings are presented in Fig. 12. Comparison of (Fig. 10) and (Fig. 12) shows that in these models of waveguides exposes more properties of band pass filters. The stop band moves to the higher frequencies approximately $10 \mathrm{GHz}$, when the structure of waveguide consists of $n$-GaAs semiconductor core and $n$-InAs ${ }_{1-x} \mathrm{Sb}_{x}$-air, TM-15 external layers.

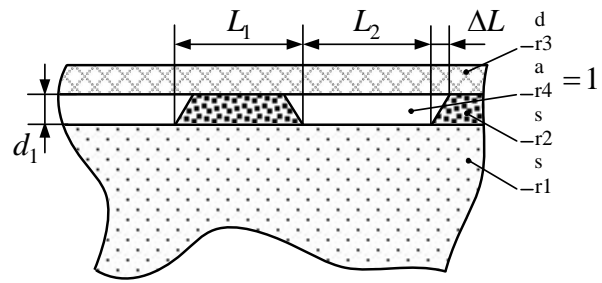

Fig. 11. The longitudinal-view of models of periodical semiconductor-dielectric waveguides, when edges of semiconductor rings are conical.

The attenuation of EM microwaves is lower than $-10 \mathrm{~dB}$ in frequency range from $23.5 \mathrm{GHz}$ to $55 \mathrm{GHz}$, when structure of waveguide consist of $n$-SiGe core and $n$-InSbair, TM-15 layers. However the lowest attenuation in this frequency range could be received till $-3 \mathrm{~dB}$, when the structure of periodical waveguide consist of $n$-SiGe core and $n$-InSb -air, TM-15 layers.

The frequency range in which the attenuation of EM 
microwaves is lower than $-10 \mathrm{~dB}$ is from $25 \mathrm{GHz}$ to $54 \mathrm{GHz}$. The lowest attenuation of EM microwave could be $-5 \mathrm{~dB}$ in this frequency range, when the structure of periodical waveguides consist of $n$-GaAs core and $n$-InAs ${ }_{1-x} \mathrm{Sb}_{x}$-air, TM-15 layers.

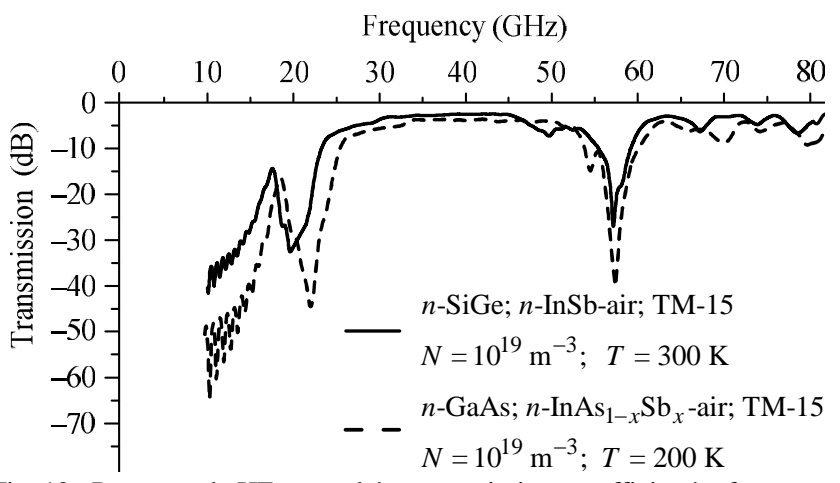

Fig. 12. Propagated $H_{11}$ mode's transmission coefficient's frequency dependences of periodical semiconductor-dielectric waveguides models, when edges of semiconductor rings are conical.

The lower attenuation of EM microwaves is received in models of waveguides which structure consist of $n$-SiGe core and $n$-InSb-air, TM-15 layers. On the other hand the control possibilities of these models by changing external temperature $T$ is lower comparing with models of waveguides which structure consist of $n$-GaAs core and $n$-InAs ${ }_{1-x} \mathrm{Sb}_{x}$-air, TM-15 layers.

The essential principles of the functioning of relevant models and application's possibilities expected to be clarified in this paper. The improvement and optimal design of selected perspective devices will be addressed in the future.

\section{CONCLUSIONS}

Comparison results of ferrite waveguide's physical experiment and numerical experiment using commercial CST Microwave Studio ${ }^{\circledR}$ software showed that ratio of transmission coefficients is about two times larger in physical experiment due to reflections from the ends of waveguide at $34.1 \mathrm{GHz}$ frequency. In both studies, the attenuation coefficients in lower frequencies varies no more then $9 \%$ and in higher frequencies $-0.1 \%$.

The lower attenuation of EM microwaves could be received in electrodynamical models of waveguides which consist of $n$-SiGe core and $n$-InSb, TM-15 layers, when density of electrons is $N=10^{19} \mathrm{~m}^{-3}$.

Frequency dependences of transmission coefficient of propagated $\mathrm{HE}_{11}$ mode in electrodynamical models of periodical semiconductor-dielectric waveguides which consist of $n$-SiGe core and $n$-InSb-air, TM-15 layers or $n$-GaAs core and $n$-InAs $\mathrm{As}_{1-x} \mathrm{Sb}_{x}$-air, TM-15 layers obtain properties of the band pass filters.

Dependences of transmission coefficient on the frequency become more smother and stop band expands in to both sides, when the edges of semiconductor rings are conical.

It has been found, that the periodical SDWs could be used in the microwave filters. The EM microwave attenuation is lower 2.2 times in model periodical waveguides which consist of $n$-SiGe core and $n$-InSb-air, TM-15 layers, than in periodical waveguides which consist of $n$-GaAs core and
$n$-InAs ${ }_{1-x} \mathrm{Sb}_{x}$-air, TM-15 layers, when density of electrons is $N=10^{19} \mathrm{~m}^{-3}$.

\section{REFERENCES}

[1] K. Istenikova, D. Faktorova, "Investigation of metamaterial structure influence on selective properties of microwave waveguide sensor", Electrical Review - Przeglad Elektrotechniczny, vol. 88, no. 7b, pp. 223-225, 2012.

[2] B. Hrycak, D. D. Czylkowski, M. Jasinski, J. Mizeraczyk, "Novel low power microwave plasma sources at atmospheric pressure", Electrical Review - Przeglad Elektrotechniczny, vol. 88, no. 8, pp. 310-312, 2012.

[3] M. D. Rotaru, J. K. Sykulski, "Dual-band electromagnetic band gap structure for noise isolation in mixed signal SiP", Electrical Review Przeglad Elektrotechniczny, vol. 86, no. 5, pp. 95-99, 2010.

[4] R. Pomarnacki, A. Krukonis, V. Urbanavicius, "Acceleration techniques for analysis of microstrip structures", Elektronika Ir Elektrotechnika, vol. 20, no. 5, pp. 108-111, 2014. [Online]. Available: http://dx.doi.org/10.5755/j01.eee.20.5.7109

[5] A. Krukonis, S. Mikucionis, V. Urbanavicius. "The influence of nonuniformity of the multi-conductor line parameters on frequency responses of the meander delay line", Elektronika Ir Elektrotechnika, vol. 19, no. 6, pp. 81-86, 2013. [Online]. Available: http://dx.doi.org/10.5755/j01.eee.19.6.4279

[6] B. J. Hu, G. Wei. "Numerical simulation of the fundamental mode of a magnetoplasma rod surrounded by a lossless dielectric layer", IEEE Trans. Plasma Science, vol. 29, no 1, pp. 1-7, 2001. [Online]. Available: http://dx.doi.org/10.1109/27.912934

[7] S. Asmontas, L. Nickelson, A. Bubnelis, R. Martavicius, J. Skudutis, "Hybrid mode dispersion characteristic dependences of cylindrical dipolar glass waveguides on temperatures", Elektronika Ir Elektrotechnika, vol. 10, no. 106, pp. 83-86, 2010.

[8] I. O. Vardiambasis, T. N. Kapetanakis, M. P. Ioannidou, "Pulse dispersion in layered cylindrical dielectric waveguides", Int. Conf. IEEE Microwaves, Communications, Antennas and Electronics Systems, 2011, pp. 1-2. [Online]. Available: http://dx.doi.org/ 10.1109/comcas.2011.6105941

[9] L. Claudepierre, N. Raveu, N Capet, "Modal analysis of anisotropic cylindrical waveguides", Microwave Conf. Proc., 2012, pp. 12891291. [Online]. Available: http://dx.doi.org/10.1109/apmc.2012. 6421898

[10] O. V. Shramkova, "Transmission spectra in ferrite-semiconductor periodic structure", Progress In Electromagnetics Research $M$, vol. 7, pp. 71-85, 2009. [Online]. Available: http://dx.doi.org/ 10.2528/PIERM09041305

[11] A. S. Kindyaka, A. D. Boardmanb, V. V. Kindyakc, "Surface magnetostatic spin wave envelope solitons in ferrite semiconductor structure", Journal of Magnetism and Magnetic Materials, vol. 253, no. 1 , pp. 8-14, 2002. [Online]. Available: http://dx.doi.org/10.1016/ S0304-8853(01)00195-0

[12] V. Kesari, P. K. Jain, B. N. Basu, "Modelling of axially periodic circular waveguide with combined dielectric and metal loading", Journal of Physics D, vol. 38, no. 18, pp. 3523-3529, 2005. [Online]. Available: http://dx.doi.org/10.1088/0022-3727/38/18/030

[13] D. Chao-Hai, L. Pu-Kun, Z. X. Qian, "Periodicity-suppressing effect of periodic lossy-dielectric-loaded cylindrical waveguide", Chinese Physics B, vol. 19, no. 4, pp. 1-6, 2010. [Online]. Available: http://dx.doi.org/10.1088/1674-1056/19/4/048703

[14] X. Qian-zhong, D. Chao-Hai, L. Pu-Kun, W. Muing-Hong, "Modes in lossy dielectric-loaded metal cylindrical waveguide for gyrotrontraveling-wave amplifier applications", in Proc. IEEE Int. Vacuum Electronics Conf., 2009, pp. 475-476.

[15] L. Nickelson, S. Asmontas, V. Malisauskas, V. Sugurovas, Open Cylindrical Gyrotropic Waveguides. Vilnius: Technika, 2007.

[16] D. Plonis, Research and Applications of Gyrotropic Devices Models. Ph.D. dissertation, Vilnius, Technika, 2014.

[17] CST Microwave Studio. CST - Computer Simulation Technology. [Online] Available: http:// www.cst.com.

[18] V. Malisauskas, D. Plonis, "The investigation of gyroelectric n-InAs phase shifters characteristics", Elektronika Ir Elektrotechnika, vol. 122, no. 6, pp. 121-124, 2012. [Online]. Available: http://dx.doi.org/10.5755/j01.eee.122.6.1836

[19] A. Dargys, J. Kundrotas, Handbook on Physical Properties of Ge, Si, GaAs and InP. Vilnius, Science and Encyclopedia Publishers, 1994.

[20] M. E. Levinshtein, S. L. Rumyantsev, M. S. Shur. Properties of advanced semiconductor materials-GaN, AIN, InN, BN, SiC, SiGe. John Wiley \& Sons, Inc.: New York, 2001. 